\title{
Toward Chemically Resolved Computer Simulations of Dynamics and Remodeling of Biological Membranes
}

\author{
Thereza A. Soares, ${ }^{\dagger}$ Stefano Vanni, ${ }^{\ddagger}$ Giuseppe Milano, ${ }^{\S}$ and Michele Cascella*,ll ${ }^{*}$ \\ ${ }^{\dagger}$ Department of Fundamental Chemistry, Federal University of Pernambuco, Cidade Universitária, Recife PE 50740-560, Brazil \\ ${ }^{\star}$ Department of Biology, University of Fribourg, 1700 Fribourg, Switzerland \\ ${ }^{\S}$ Dipartimento di Chimica e Biologia, Università di Salerno, Via Giovanni Paolo II, 132, I-84084 Fisciano, Italy \\ "Department of Chemistry and Centre for Theoretical and Computational Chemistry (CTCC), Sem Saelands vei 26, 0371 Oslo, \\ Norway
}

\begin{abstract}
Cellular membranes are fundamental constituents of living organisms. Apart from defining the boundaries of the cells, they are involved in a wide range of biological functions, associated with both their structural and the dynamical properties. Biomembranes can undergo large-scale transformations when subject to specific environmental changes, including gel-liquid phase transitions, change of aggregation structure, formation of microtubules, or rupture into vesicles. All of these processes are dependent on a delicate interplay between intermolecular forces, molecular crowding, and entropy, and their understanding requires approaches that are able to capture and rationalize the details of all of the involved interactions. Molecular dynamics-based computational models at atom-level resolution are, in principle, the best way to perform such investigations. Unfortunately, the relevant spatial and time dimensionalities involved in membrane remodeling phenomena would require computational costs that are today

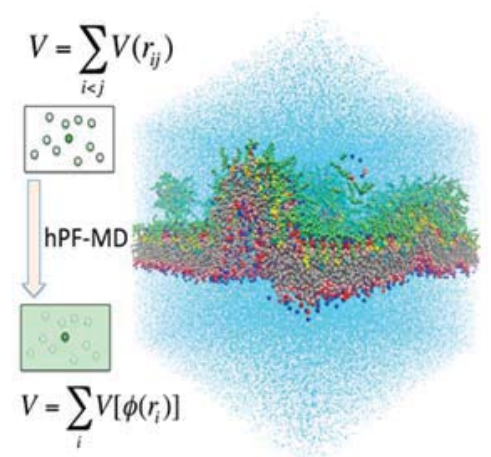
unaffordable on a routinely basis. Such hurdles can be removed by coarse-graining the representations of the individual molecular components of the systems. This procedure anyway reduces the possibility of describing the chemical variations in the lipid mixtures composing biological membranes. New hybrid particle field multiscale approaches offer today a promising alternative to the more traditional particle-based simulations methods. By combining chemically distinguishable molecular representations with mesoscale-based computationally affordable potentials, they appear as one of the most promising ways to keep an accurate description of the chemical complexity of biological membranes and, at the same time, cover the required scales to describe remodeling events.
\end{abstract}

The present limits of applicability of computer modeling are being continuously expanded by both the steady increase of computational power and, in parallel, the continuous development of new modeling approaches and software. Thanks to such advances, today the molecular modeling community is taking up the challenge of unraveling the molecular details at the base of complex phenomena in the condensed phase occurring at intrinsically large time and length scales.

The dynamical behavior of water-lipid mixtures is a prototypical case of such phenomena. The structure and organization of lipids in water solutions is in fact dependent on several factors, including the chemical composition of the species or their relative concentration, as well as external thermodynamic conditions like temperature or pressure. The properties of such mixtures are strictly collective, meaning that they appear only in the presence of a large number of crowding and self-assembling molecules. Therefore, their properties cannot be deduced from the determination of any physical-chemical feature of the individual components nor can their study be reducible to sizes smaller than some characteristic values. ${ }^{1}$

Biological membranes are a special case of lipid mixtures occurring in living organisms. Their modeling is particularly difficult because of their complex chemical composition, ${ }^{2}$ further complicated by the presence or both membrane-integral and membrane-peripheral proteins ${ }^{3}$ and by the fact that bioactivity is often associated with dynamical structural deformations, like the appearance of rafts, patches, undulations, tubules, and so forth. ${ }^{4,5}$ The description of such features would require the implementation of intrinsically large models (characteristic lengths on the order $\left.\sim 10-10^{3} \mathrm{~nm}\right)$ simulated for very long times $\left(\sim 10^{-6}-10^{-3} \mathrm{~s}\right){ }^{6,7}$

Today, we are on the verge of moving from an early pioneering phase, where biomembranes are usually represented by flat periodic bilayers with model lipid compositions, into more mature times where both the biomembrane structure and dynamics can be represented close to their realistic complexity. This progress is the consequence of developments in multiscale modeling methods that allow exploitation of the advantages of representations at low resolution (like the use of smooth potentials and the need for a reduced number of variables) in combination with those at higher resolution (like chemical accuracy). ${ }^{8-10}$

In this Perspective, after briefly recalling the main structural and dynamical features of biomembranes, we provide a brief 
Today, we are on the verge of moving from an early pioneering phase, where biomembranes are usually represented by flat periodic bilayers with model lipid compositions, into more mature times where both the biomembrane structure and dynamics can be represented close to their realistic complexity

overview of what is feasible today in multiscale modeling of such systems. We finally highlight some advances in hybrid particle/mesoscale modeling and some of its most recent applications to membrane dynamics. A series of studies on multiphase systems formed by few components are today present in the literature, demonstrating the reliability of this modeling approach to soft-matter problems. Working at chemical molecular resolution, this relatively new methodology is paving a very promising way toward accurate simulations of realistic models of biomembranes at relatively cheap computational costs.

Polymorphism in Biological Membranes. Biological membranes delimit cellular and intracellular compartments where biomolecules are spatially harbored, and where timely coordination of thousands of complex biochemical reactions take place. Biological membranes are not static but dynamic surfaces undergoing structural changes in response to intrinsic or extrinsic forces. ${ }^{11-13}$ In eukaryotes, membrane proteins and lipids are internalized, externalized, or transported within cells via their insertion in the membrane as small vesicle or thin tubules, or by uptake by lipid transport proteins rather than by bulk diffusion of single molecules. The disordered liquid-crystalline state characteristic of biological membranes allows lipid diffusion and mix in the bilayer leaflet plane through mostly non-Brownian (anomalous) diffusion. ${ }^{14}$ Because biological membranes are continuously revolved by trafficking intermediates such as vesicles and tubules, the origination and stabilization of high-curvature compartments become crucial for life.

The generation of membrane curvature is a precondition for membrane shaping in living organisms. ${ }^{1,15}$ It has been extensively investigated in eukaryotes, though much less is known for prokaryotes. ${ }^{16,17}$ Membrane curvature establishes cellular and subcellular architecture in living organisms while regulating signal and molecular traffic across biological membranes. It allows for the remarkable array of shapes in cellular organelles (e.g., the tubular endoplasmic reticulum, the saccularfenestrated Golgi complex), expanding their membrane surface area for reactions through the shaping of folds and tubules. It also modulates membrane trafficking through bending, invagination, protrusion, fusion, fission, and vesicle budding. A recent experiment combining stretch stress and fluorescent imaging has recently demonstrated that readaptation of membranes after a mechanical deformation occurs via purely passive physical principles. ${ }^{18}$ Rapid progress has been made in expanding membrane curvature research beyond membrane trafficking to organelle architecture. The new view of membrane curvature as an active means to control the spatial organization and activity of cells has replaced the old view of it as representing merely a passive geometric feature of biological membranes. ${ }^{19,20}$
Understanding Membrane Deformation by Computer Modeling. Cellular membranes are characterized by a large diversity of lipids. ${ }^{2}$ Such a complexity stems from the numerous possible combinations that can be obtained as a consequence of the variations in both their hydrophobic tails (e.g., saturated vs unsaturated vs polyunsaturated fatty acids) and their polar moieties (e.g., phoshocholine, phosphoethanolamine, phosphoinositides, ...). Hence, a detailed chemical understanding of how lipid composition may play an important role in driving cellular processes is paramount to correctly identify the contribution of lipid membrane properties in biological phenomena.

Investigating the molecular properties of membranes with subnanometer resolution, however, has remained extremely challenging due to inherent limitations of the available experimental methodologies, like the limited system sizes accessible by NMR, the intrinsic single-configuration data coming from $\mathrm{X}$-ray scattering, or the relatively low resolution from electron microscopy. ${ }^{21,22}$ To overcome these limitations, particle-based computational approaches, namely, molecular dynamics (MD) simulations, have emerged in the last 2 decades as an irreplaceable tool to gain a detailed structural understanding of lipid membranes.

All-Atom (AA) Models. Atomistic MD simulations represent chemical systems using classical Newtonian dynamics laws. The potential energy of the systems is written as a sum of effective interactions among the atoms that compose the molecular system of interest. These potential energy terms mimic the effect of the quantum-mechanical interactions among nuclei and electrons gluing the atoms into higher-order (inter)molecular structures. The most typical form of such a potential for biomolecules takes the following form:

$$
\begin{aligned}
V= & \sum_{\text {bonds }} \frac{1}{2} k_{\mathrm{b}}\left(r-r_{0}\right)^{2}+\sum_{\text {bends }} \frac{1}{2} k_{\mathrm{a}}\left(\theta-\theta_{0}\right)^{2} \\
& +\sum_{\text {torsions }} k_{\mathrm{t}}(\cos n \phi+1)+\sum_{i<j} \frac{1}{4 \pi \epsilon_{0}} \frac{q_{i} q_{j}}{r_{i j}} \\
& +\sum_{i<j}\left(\frac{A}{r^{12}}-\frac{B}{r^{6}}\right)
\end{aligned}
$$

Here, the first three terms take into account the interactions among bonded atoms (usually called bonded interactions), which are responsible for the appearance of a defined molecular structure and for its local rigidity. The last two terms account for long-range interactions (usually called nonbonded interactions), which are represented as a combination of Coulomb and van der Waals terms. This mechanical model requires the definition of a large set of parameters like the force constants and the equilibrium values in the bonded interactions, the electrostatic charges assigned to each atom, and the Lennard-Jones coefficients for each atom-type couple. In the literature, there exist different sets of parameters, which are obtained from a combination of different experimental measures and/or computational models, whose reliability may be dependent on not only the implementation per se but also the interface with pre-existing models for water or ions. ${ }^{23-30}$ For a critical assessment of the force fields available today for lipid simulations, see, for example, refs 22,31 , and 32 .

The computational costs of atomistic MD simulations are dependent on two major aspects. The first is the high frequency of the vibrational modes, which impose the use of relatively short time steps for numerical integration of the equations of 
motion $(\sim \mathrm{fs})$. The second is the slow decay of the nonbonded terms, which in principle requires growth of operations proportional to $N^{2}$, increasing the size of the systems under study ( $N$ being the total number of atoms). Even though different algorithms to avoid such strict bottlenecks are available today, the combination of these problems severely limits any routine access to the dimensions and characteristic times relevant to investigate membrane curvature problems.

In fact, although recent pioneering studies have allowed the investigation of complex and biologically relevant model membranes, ${ }^{33}$ using atomistic models, MD simulations of membranes at such a level of resolution are usually limited to small model systems, typically flat bilayers consisting of few hundreds of the same lipid type.

Since the first pioneering attempts to investigate the mechanism of membrane curvature formation by BAR domains, ${ }^{34,35}$ attempts to investigate membrane curvature using atomistic $\mathrm{MD}$ simulations have become more and more common. ${ }^{36-41}$

All of these studies, however, are hampered by inevitable limitations for what pertains to the sizes that can be investigated using atomistic simulations. Specifically, any deformation of the lipid bilayer will occupy a characteristic volume that needs to be fully included into the simulation box. For example, for a relatively simple membrane undulation, both the length of the simulation box normal to the lipid bilayer plane and the membrane axis along which the undulation occurs need to be on the order of at least $20 \mathrm{~nm}$. In these conditions, with the possible exception of dedicated hardware, it is very difficult to go beyond tens of nanoseconds per day, even when using state-of-the-art hardware and software.

At the same time, such sizes are relatively small as compared to those required to investigate membrane remodeling and vesiculation. In fact, the smallest biological vesicles show diameters of approximately $40-50 \mathrm{~nm},{ }^{42}$ implying that simulations boxes for biologically relevant systems may easily reach lengths on the order of $100 \mathrm{~nm}$ or more.

In addition, the use of periodic boundary conditions imposes an additional topological restraint on extended bilayers, forcing the presence of both positive and negative curvatures within the length of the simulation box. This significantly reduces the size of membrane patches with specific curvature that can be simulated and analyzed.

Coarse-Grained Models. Coarse-grained (CG) modeling is a very general term that includes all models based on a procedure of mapping a complex system dependent on a number $N$ (typically very large) of variables into a simplified one formed by a smaller number of variables $M<N$ (Figure 1 ). In a consequent renormalization procedure, the total energy of the system is defined through a Hamiltonian

$$
H^{\prime}(M) \neq H(N)
$$

While $H^{\prime}$ is necessarily different from that of the original system, if properly mapped, then the statistical behavior of the complete and reduced systems will match each other. This implies that both representations will yield similar expectation values for the observables of interest

$$
\begin{gathered}
\left\langle O_{M}\right\rangle=\frac{1}{Z_{M}} \int O(M) \mathrm{e}^{-\beta H^{\prime}(M)} \mathrm{d} \Gamma_{M} \\
\approx \frac{1}{Z_{N}} \int O(N) \mathrm{e}^{-\beta H(N)} \mathrm{d} \Gamma_{N}=\langle O\rangle_{N}
\end{gathered}
$$
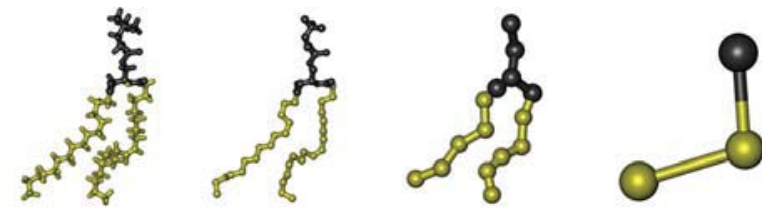

All-atom

United-atom

Coarse Grained

Coarse Grained

(low resolution)

Figure 1. Molecular models of lipids. Usual representations of phospholipids used in molecule-based computer simulations, from an all-atom-detailed picture (left) to coarser representations still capturing chemical details to low-resolution coarse models containing a minimal number of bodies (right). The hydrophobic regions are colored in yellow, the hydrophilic head is colored in dark gray.

where $O$ is a generic physical observable, its expectation value $\langle O\rangle$ is defined through the corresponding ensemble average, and $Z_{N}$ and $Z_{M}$ are the partition functions

$$
\begin{aligned}
& Z_{N}(T)=\int \mathrm{e}^{-\beta H(N)} \mathrm{d} \Gamma_{N} \\
& Z_{M}(T)=\int \mathrm{e}^{-\beta H^{\prime}(M)} \mathrm{d} \Gamma_{M}
\end{aligned}
$$

The mapping system will profit from reduced computational costs due to the reduction of the number of degrees of freedom. On the other hand, the consequent contraction of the phase space will produce an intrinsic acceleration of the events. This, on the one hand, guarantees an even further acceleration in the sampling; on the other hand, events in CG-MD simulations will occur on a time scale that cannot be straightforwardly connected to the real one.

In CG modeling, the mapping procedure is not universally defined; in fact, the same molecular system may be represented at different levels of resolution, typically using centroids called beads to represent groups of atoms, and by using different analytical forms for the respective potential energy. ${ }^{10}$ For lipids, the most common CG models today adopt quasi-atomistic resolutions and use two-body interaction potentials to represent the hydrophobic/hydrophilic behavior of the lipid polar heads and apolar tails (Figure 1)..$^{43-47}$

The investigation of membrane remodeling processes by means of CG-MD simulations was pioneered by studies that either used very coarse representations ${ }^{48}$ or addressed relatively small systems with extreme curvature values. ${ }^{49}$ More recently, CG-MD simulations with chemical-level detail have been used to investigate systematically the role of membrane curvature on membrane molecular properties, ${ }^{50}$ for example, determining how gel-phase lipid membranes soften upon bending, thus helping the localization of the curvature. ${ }^{51}$ Simulations of curved structures (vesicles, tubules) with dimensions comparable with those found in the cell have become computationally tractable using coarser models ${ }^{52}$ or chemically accurate CG models, thus allowing one to investigate the interplay between more realistic and biologically relevant lipid composition and membrane geometry. ${ }^{53,54}$

In parallel to investigating how membrane curvature modulates membrane structural properties, MD simulations have been also used to explicitly investigate membrane remodeling events, namely, bending, fusion, and fission, while retaining chemical specificity (Figure 2). These processes have been reviewed elsewhere. ${ }^{6,56}$ Here we stress how notable efforts have been focusing on understanding the explicit role of 


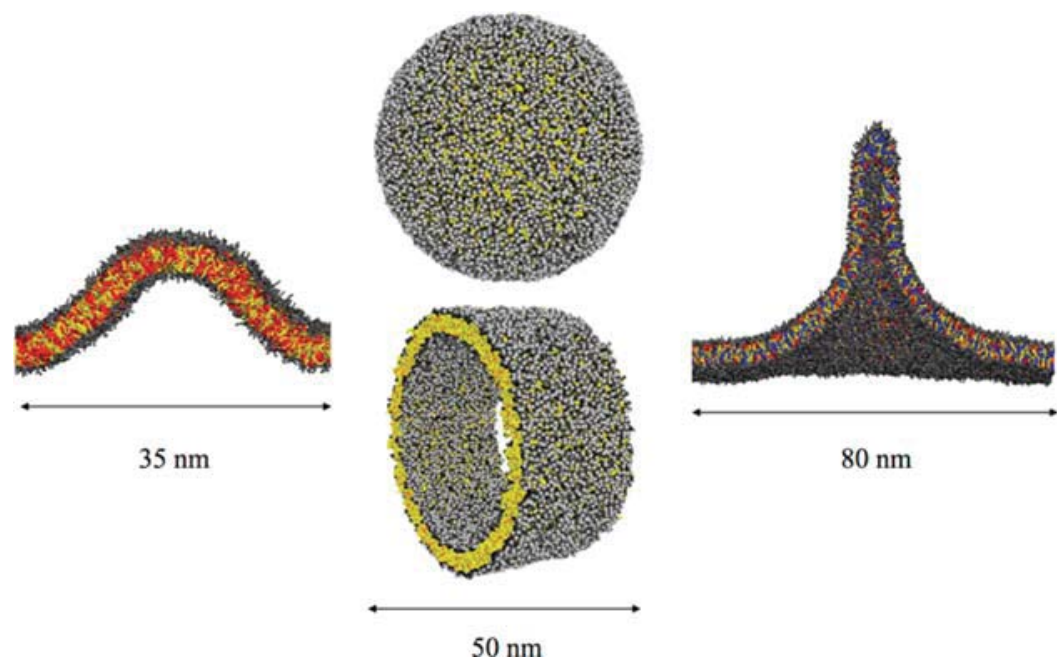

Figure 2. Examples of modeling of membrane curvature types. Different features can appear at different length scales. (left) Buckled membrane; (center) lipid vesicle or tubule; (right) membrane tubule formation by force pulling. Lipid polar heads are in gray, and hydrophobic tails are in yellow, red, or blue, depending on their chemical properties. Systems in the figure were taken from refs 53 and 55.

membrane curvature and lipid composition in both membrane fusion $^{57,58}$ and fission. ${ }^{55}$ These studies have further underlined the importance of retaining chemical accuracy when studying curvature-dependent phenomena in membrane processes. For example, membrane fusion depends on the ratio between phosphatidyl-choline and phosphatidyl-ethanolamine, ${ }^{57}$ while membrane fission propensity is strongly affected by membrane elasticity. ${ }^{55}$

Hybrid Particle-Continuum Approaches. However simplified, CG approaches based on particle reduction are still subject to the same computational bottlenecks of AA models, like the need to evaluate distance-dependent intermolecular interactions, or a relatively intense communication among nodes in parallel architectures. As a consequence, unless massively parallel architectures are available, common CG simulations are still limited to systems with sizes not larger than $100 \mathrm{~nm}$ in cell length and with relatively short sampling.

\section{Unless massively parallel archi- tectures are available, common CG simulations are still limited to systems with sizes not larger than $100 \mathrm{~nm}$ in cell length and with relatively short sampling.}

Continuum representations are not bound to the limits of particle-based models and thus can be used at the scales of relevance for biomembrane reshaping phenomena. Among several proposed approaches, self-consistent field (SCF) theory has been extensively developed and applied to soft matter modeling. SCF theory describes the model systems by using density fields; the mutual interactions between molecular segments is modeled by an effective potential involving these segments and static external fields. ${ }^{59}$ Several applications to block copolymers, ${ }^{60,61}$ colloidal particles, ${ }^{62}$ and simplified models of proteins ${ }^{63}$ have already shown that the SCF theory is a useful and powerful approach.

The main drawback of these models is the limitation to very simple molecular architectures, typically linear Gaussian chains. This problem can be circumvented by including hybrid approaches based on a discrete representation of the components coupled to a continuous field representation determining the interactions. Early examples of such an approach were proposed by Kawakatsu and Kawasaki for dynamical models of discrete surfactant molecules embedded in a continuous field representing an immiscible binary mixture. ${ }^{64-66}$ Similarly, Laradji et al. presented Monte Carlo simulations of polymer brushes where density fields were calculated on the fly from discrete particle positions. ${ }^{67}$ About 10 years ago, Daoulas and Müller introduced a Monte Carlo single chain in mean field approach, where the calculations of the density fields could be performed on a grid. This approach has been successfully applied to block copolymer systems and homopolymers. ${ }^{68,69}$ More recently, a hybrid scheme for MD compatible with both atomistic force fields and/or specific CG models (Hybrid Particle Field MD, hPF-MD hereafter) was introduced and extensively validated for different molecular models, ${ }^{70-72}$ including molecular surfactants, atomistic models of polymers, and biomembranes. ${ }^{73-76}$

hPF-MD Theory. The many-body problem associated with determination of the state of a molecular system is regarded as a problem of derivation of the partition function of a single independent molecule in an external potential $V(\mathbf{r})$. Such a potential takes accounts of the effective action of all other particles present in the system to the particle of interest. The functional form of the density-dependent total energy $W$ needs to be assumed, and a common form is the one reported below

$$
\begin{aligned}
W & {\left[\left\{\phi_{k}(\mathbf{r})\right\}\right] } \\
& =\int \mathrm{d} \mathbf{r}\left(\frac{1}{2 \beta} \sum_{k k^{\prime}} \chi_{k k^{\prime}} \phi_{k}(\mathbf{r}) \phi_{k^{\prime}}(\mathbf{r})+\frac{1}{2 \kappa}\left(\sum_{k} \phi_{k}(\mathbf{r})-\phi_{0}\right)\right)^{2}
\end{aligned}
$$

where $\phi_{k}(\mathbf{r})$ is the number density of the $k$ th species (or atom type) present in the system at position $\mathbf{r}$ and $\chi_{k k^{\prime}}$ are the mean field parameters for the interaction of a particle of type $k$ with the density field generated by particles of type $k^{\prime}$. Thus, any given particle interacts with the surrounding through a density field, rather than through direct pairwise interactions. It can be shown, in the framework of SCF theory, that the external 
potential representing the effect of surrounding particles is given by

$$
V_{k}(\mathbf{r})=\frac{\delta W}{\delta \phi_{k}(\mathbf{r})}=\frac{1}{\beta} \sum_{k^{\prime}} \chi_{k k^{\prime}} \phi_{k^{\prime}}(\mathbf{r})+\frac{1}{\kappa}\left(\sum_{k} \phi_{k}(\mathbf{r})-\phi_{0}\right)
$$

The derivation of a functional form for the effective potential acting on each particle allows determination of the forces and thus for the integration of the equations of motion following standard MD algorithms. More details about the implementation of this approach, and a complete derivation of eq 6, is reported elsewhere."

Hybrid Particle Field MD Studies on Lipid-Water Mixtures. So far, hPF-MD studies on lipid-water mixtures have addressed relatively simple two- or three-component systems. Pertinent to this discussion, these initial studies addressed the capability of hPF-MD models to reproduce both the density profiles of different bilayers formed by lipids of biological interest and the lamellar versus nonlamellar phases-a crucial test for lipid models that aim at investigating membrane morphology. ${ }^{9}$ In particular, Milano and co-workers showed how hPF-MD predicts different aggregation states at increasing concentrations of dipalmitoylphosphatidylcholine (DPPC) in water. For example, their simulations yielded reverse micellar hexagonal, flat bilayer, single bicelle, and micellar phases. ${ }^{74}$

In the past months, Milano and co-workers introduced hydrodynamic correlation into the hPF-MD equations. ${ }^{77}$ This innovation brings hPF-MD simulations beyond the mean-field limit and in perspective will improve the applicability of the method to study phenomena that do not occur strictly under diffusive limit conditions. ${ }^{77}$ Of importance here, authors presented hPF-MD data of a fully solvated lipid vesicle composed of 4055 DPPC molecules, simulated on a single processor on a microsecond time scale (Figure $3 \mathrm{~A}$ ).
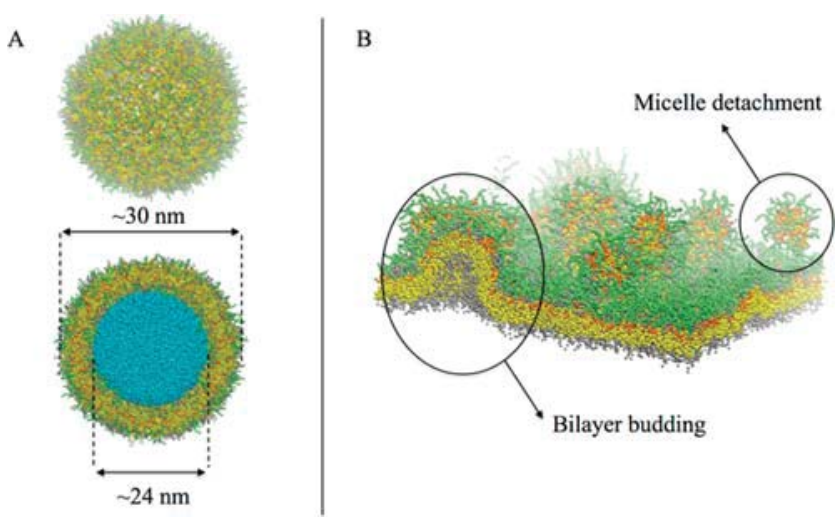

Figure 3. Examples of hPF-MD studies on curved membrane systems. (A) Lipid vesicle composed of 4055 DPPC molecules (depicted in yellow and gray) interacting with 1745 molecules of TX-100 (green and orange). The bottom panel shows a cross section of the vesicle. The water particles in the inner pocket are shown as blue beads. (B) Structural distortion and initial solubilization of a flat bilayer upon interaction with an excess of TX-100.

hPF-MD calculations are not restricted to single-processor tasks. In fact, they can very easily and very efficiently parallelize, showing scalability performances well beyond those of traditional MD. ${ }^{72,9}$ A very recent study by Milano and co-workers provides an excellent example of the efficiency of parallel hPF-MD simulations to describe membrane morphology changes
(Pizzirusso et al. Biomembrane solubilization mechanism by Triton X-100: A computational study of the three stage model, submitted, unpublished result). This work investigated deformations of the DPPC bilayer structure due to interference with Triton X-100 (polyethylene glycol) detergent. hPF-MD simulations could provide a very detailed description of the different perturbations to the bilayer organization occurring at different concentrations of Triton X-100, from local distortions of the shape of the lipid aggregates to complete membrane solubilization (Figure 3B). This study also investigated the differences in the solubilization mechanism occurring in flat bilayers or in highly curved vesicles.

Thanks to the computationally efficient methodology, authors could test 30 different initial conditions, including the relative concentration of the surfactant, its initial localization in the system, and the radius of curvature of the vesicle. The study could show how the flipping times of the surfactant are correlated to the radius of curvature of the membrane bilayers. Moreover, it could be possible to capture how the entropy-driven preference of positive curvature by Triton induces budding deformations in flat bilayers, promoting detachment of smaller vesicle, which rapidly evolve into completely solubilized $\mathrm{mi} /$ bicelles.

Despite quite extensive exploration over a large number of different starting conditions, the computational costs for the study have been relatively low. In fact, the work could be conducted on a small-sized parallel architecture using the hPF-MD implementation in the OCCAM code. Details concerning the OCCAM code used to perform the hPF-MD simulations are reported in refs 9 and 72 .

Final Remarks and Perspective. In a 2006 article in Physics Today, Phillips and Quake showed how the nanoscale is a peculiar dimensionality at which very different types of energies (chemical, mechanical, or electrostatic) appear all to be of the same order of magnitude as the thermal one. ${ }^{78}$ As a consequence, all phenomena that are driven by interactions occurring at that scale are determined by the very subtle balancing among all contributions to the enthalpy and by the action of the entropy. In other words, the mesoscopic behavior of molecular systems may be not only quantitatively but also qualitatively affected by apparently minor changes in the chemistry of each individual molecular component, as well as in the thermodynamic conditions applied.

\section{The mesoscopic behavior of mo- lecular systems may be qualita- tively affected by apparently minor changes in the chemistry of each individual molecular component.}

From a computational perspective, it would be thus expected that all of these ingredients were properly included and treated in the models of choice. Specifically to membranes, an ideal modeling approach would be able to handle mesoscopic dimensionalities (for example, simulation boxes of $\sim 10^{2-3} \mathrm{~nm}$ order length) for sufficiently long simulation times (at least $\sim 10^{-6}-10^{-3} \mathrm{~s}$ ), to distinguish the chemical variability of the components, and to include entropic effects also due to the solvent.

Atomic or quasi-atomic resolution models with explicit solvation are the only ones that guarantee a correct, or anyway sufficiently 
accurate, description of the chemistry, as well as of the entropy contributions. Nonetheless, as previously discussed, they are affected by intrinsically high computational costs that prevent their systematic use on a mesoscopic scale. Therefore, multiscale modeling strategies combining molecular-level and simplified resolutions appear as a more promising route toward accurate simulations of very large and chemically complex systems.

\section{Multiscale modeling strategies combining molecular-level and simplified resolutions appear as a more promising route toward accurate simulations of very large and chemically complex systems.}

From the experimental point of view, the advances in timeresolved spectroscopy and the development of more powerful radiation sources allow for more and more accurate structural and dynamical data on the evolution of lipid bilayers under external chemical or mechanical stimuli (i.e., see ref 79). It is easily foreseen that such experimental studies on model bilayers are going to produce accurate biological data on in vivo membranes in the near future. In fact, an exciting first structural image of a biomembrane obtained by H/D labeling and neutron scattering has just been published. ${ }^{80}$

Future computational studies on remodeling of biological membranes will necessarily need to take into account the role of both peripheral and integral proteins embedded into the system. Any accurate description of membrane/protein interactions will thus require adequate treatment of the electrostatic interactions between the different lipids and the polypeptide chains, which, apart from the strongly polar nature of their backbone, may be often characterized by electrolytic side-chains.

Among the various multiscaling techniques available today, we evidenced here how hPF-MD is emerging as a methodology that may play a crucial role in the study of membrane dynamics in the near future. Very important recent developments both from the methodological and implementation points of view are worth mentioning. These consist of (i) derivation of a pressure coupling algorithm, ${ }^{71}$ (ii) explicit treatment of electrostatic interactions within particle/field, ${ }^{81}$ and (iii) the previously mentioned inclusion of hydrodynamics to treat correlated motion of particles. ${ }^{77}$ Moreover, we welcome the appearance of new hybrid particle/field software implementations for both CPU- $^{72}$ and GPU-based ${ }^{82}$ architectures as key steps toward the popularization of such an approach in the modeling community.

So far, studies from the literature showed how hPF-MD can efficiently address systems composed of several thousands of lipids to observe complex dynamical processes, requiring access to commodity cluster facilities (tens to a few hundreds of CPUs) or even by running on serial tasks. hPF-MD parameters for the most common phospholipids and triglycerides are already available in the literature. Likely, the hPF-MD models for other important components of biomembranes will appear in the near future. Incorporation into the hPF-MD formalism of CG models of proteins with accurate electrostatics, for example, like those developed in the past by some of us, ${ }^{83-85}$ will constitute the next crucial step toward an accurate computational description of morphological changes of biomembranes at chemical resolution.

hPF-MD studies have so far explored systems with a relatively easy composition; therefore, its reliability in treating complex mixtures still requires some strict assessment. Anyway, it appears that exploration of the complex behavior of realistic models of biological lipid mixtures using such methodology is at hand.

\section{AUTHOR INFORMATION}

\section{Corresponding Author}

*E-mail: michele.cascella@kjemi.uio.no.

\section{ORCID}

Michele Cascella: 0000-0003-2266-5399

\section{Funding}

T.A.S.: Brazilian funding agencies FACEPE (APQ-0732-1.06/ 14) and CAPES (BioComp 23038.004630/2014-35) and the Swedish funding agency STINT (IG2011-2048). S.V.: Swiss National Science Foundation (Grant No. PP00P3 163966). M.C.: Norwegian Research Council through the CoE Centre for Theoretical and Computational Chemistry (CTCC) Grants $179568 / \mathrm{V} 30$ and $171185 / \mathrm{V} 30$.

\section{Notes}

The authors declare no competing financial interest.

\section{Biographies}

Thereza A. Soares received her Ph.D. in Chemistry from Federal University of Pernambuco in 2001 after academic terms at The Scripps Research Institute, the University of Houston, and Pacific Northwest National Laboratory. In 2005, after a postdoctoral appointment at the Swiss Federal Institute of Technology in Zurich and in Lausanne, she joined the Pacific Northwest National Laboratory research staff. Since 2010, she has been a professor of chemistry at Federal University of Pernambuco and has held appointments as guest professor at the Swiss Federal Institute of Technology in Lausanne and Umeå University. Her research interests are the development and application of computational methodologies with the aim of understanding biomolecular processes and to interpret experimental observations.

Stefano Vanni studied Physics at the University of Bologna, Italy, where he received his B.Sc and M.Sc in Theoretical Physics. Later, he obtained his Ph.D. in the field of Computational Chemistry and Biochemistry at EPFL, Lausanne, before joining the Institut de Pharmacologie Cellulaire et Moleculaire at CNRS in Sophia Antipolis, France, as a postdoctoral fellow. Since 2015, he has been a tenured researcher at CNRS, and since 2017, he has been a Swiss National Science Foundation Associate Professor in the Department of Biology at the University of Fribourg, Switzerland. His main research focus is molecular understanding of the role of membrane and lipid physicochemical properties in intracellular trafficking processes and lipid metabolism.

Giuseppe Milano is associate professor at the Department of Chemistry and Biology, University of Salerno, Italy. He got his degree in theoretical chemistry in 1995 and a Ph.D. in macromolecular chemistry. He has been guest scientist at the Theory Group of Max Planck Institute for Polymer Research in 2000, in 2003, he was an Alexander Von Humboldt fellow at the International University Bremen, and in 2008, he was an invited fellow of the Japan Society for Promotion of Science at Tohoku University. His research interests are simulation methods, with particular interest devoted to development of methods for modeling soft matter across time and length scales.

Michele Cascella graduated in Chemistry from the University La Sapienza in Rome and got a Ph.D. in Statistical and Biological Physics at the International School for Advanced Studies, Trieste in 2004. After a postdoctoral experience at the Ecole Polytechnique Féderale de Lausanne, where he worked on electron carriers and biological electron transfer, in 2008, he began his independent researcher career as Swiss National Science Foundation Professor in the Department of Chemistry and Biochemistry of the University of Bern. Since 2014, he has been an 
Associate Professor at the University of Oslo. His research focuses on multiscale models of biochemical processes using multiresolution schemes (QM/MM/CG/mesoscale). In November 2017, he became one of the lead scientists of the newly founded Norwegian Centre of Excellence - Hylleraas Centre for Quantum Molecular Sciences.

\section{ACKNOWLEDGMENTS}

T.A.S. was the recipient of a guest professorship from the Umea Centre for Microbial Research (UCMR) Linnaeus Program. The authors acknowledge the Centre for Theoretical and Computational Chemistry (CTCC) for facilitating meetings and exchange of ideas.

\section{REFERENCES}

(1) Jacobson, K.; Mouritsen, O. G.; Anderson, R. G. W. Lipid rafts: at a crossroad between cell biology and physics. Nat. Cell Biol. 2007, 9, 7-14.

(2) van Meer, G.; Voelker, D. R.; Feigenson, G. W. Membrane lipids: where they are and how they behave. Nat. Rev. Mol. Cell Biol. 2008, 9, $112-124$.

(3) Engelman, D. M. Membranes are more mosaic than fluid. Nature 2005, 438, 578-580.

(4) Bigay, J.; Antonny, B. Curvature, Lipid Packing, and Electrostatics of Membrane Organelles: Defining Cellular Territories in Determining Specificity. Dev. Cell 2012, 23, 886-895.

(5) Kozlov, M. M.; Campelo, F.; Liska, N.; Chernomordik, L. V.; Marrink, S. J.; McMahon, H. T. Mechanisms shaping cell membranes. Curr. Opin. Cell Biol. 2014, 29, 53-60.

(6) Marrink, S. J.; Tieleman, D. P. Perspective on the Martini model. Chem. Soc. Rev. 2013, 42, 6801-6822.

(7) Ingolfsson, H. I.; Arnarez, C.; Periole, X.; Marrink, S. J. Computational 'microscopy' of cellular membranes. J. Cell Sci. 2016, 129, 257-268.

(8) Klein, M. L.; Shinoda, W. Large-scale molecular dynamics simulations of self-assembling systems. Science 2008, 321, 798-800.

(9) Milano, G.; Kawakatsu, T.; De Nicola, A. A hybrid particle-field molecular dynamics approach: a route toward efficient coarse-grained models for biomembranes. Phys. Biol. 2013, 10, 045007.

(10) Vanni, S.; Cascella, M. In Chemical Modelling; Joswig, J.-O., Springborg, M., Eds.; Royal Society of Chemistry, 2016; Vol. 12, p 1-52.

(11) McMahon, H. T.; Gallop, J. L. Membrane curvature and mechanisms of dynamic cell membrane remodelling. Nature 2005, 438, 590-596.

(12) Parthasarathy, R.; Groves, J. T. Curvature and spatial organization in biological membranes. Soft Matter 2007, 3, 24-33.

(13) Goni, F. M. The basic structure and dynamics of cell membranes: An update of the Singer-Nicolson model. Biochim. Biophys. Acta, Biomembr. 2014, 1838, 1467-1476.

(14) Kusumi, A.; Nakada, C.; Ritchie, K.; Murase, K.; Suzuki, K.; Murakoshi, H.; Kasai, R. S.; Kondo, J.; Fujiwara, T. Paradigm shift of the plasma membrane concept from the two-dimensional continuum fluid to the partitioned fluid: High-speed single-molecule tracking of membrane molecules. Annu. Rev. Biophys. Biomol. Struct. 2005, 34, $351-354$.

(15) Zimmerberg, J.; Kozlov, M. M. How proteins produce cellular membrane curvature. Nat. Rev. Mol. Cell Biol. 2006, 7, 9-19.

(16) Schwechheimer, C.; Kuehn, M. J. Outer-membrane vesicles from Gram-negative bacteria: biogenesis and functions. Nat. Rev. Microbiol. 2015, 13, 605-619.

(17) Bohuszewicz, O.; Liu, J. W.; Low, H. H. Membrane remodelling in bacteria. J. Struct. Biol. 2016, 196, 3-14.

(18) Kosmalska, A. J.; Casares, L.; Elosegui-Artola, A.; Thottacherry, J. J.; Moreno-Vicente, R.; Gonzalez-Tarrago, V.; del Pozo, M. A.; Mayor, S.; Arroyo, M.; Navajas, D.; et al. Physical principles of membrane remodelling during cell mechanoadaptation. Nat. Commun. 2015, 6, 7292.

(19) Antonny, B. Mechanisms of Membrane Curvature Sensing. Annu. Rev. Biochem. 2011, 80, 101-123.
(20) Iversen, L.; Mathiasen, S.; Larsen, J. B.; Stamou, D. Membrane curvature bends the laws of physics and chemistry. Nat. Chem. Biol. 2015, 11, 822-825.

(21) Marsh, D. Handbook of Lipid Bilayers; Royal Society of Chemistry: London, 2013.

(22) Botan, A.; Favela-Rosales, F.; Fuchs, P. F. J.; Javanainen, M.; Kanduc, M.; Kulig, W.; Lamberg, A.; Loison, C.; Lyubartsev, A.; Miettinen, M. S.; et al. Toward Atomistic Resolution Structure of Phosphatidylcholine Headgroup and Glycerol Backbone at Different Ambient Conditions. J. Phys. Chem. B 2015, 119, 15075-15088.

(23) Brodsky, A. Is there predictive value in water computer simulations? Chem. Phys. Lett. 1996, 261, 563-568.

(24) van der Spoel, D.; van Maaren, P. J.; Berendsen, H. J. C. A systematic study of water models for molecular simulation: Derivation of water models optimized for use with a reaction field. J. Chem. Phys. 1998, 108, 10220-10230.

(25) Pandit, S. A.; Bostick, D.; Berkowitz, M. L. Molecular dynamics simulation of a dipalmitoylphosphatidylcholine bilayer with $\mathrm{NaCl}$. Biophys. J. 2003, 84, 3743-3750.

(26) Mukhopadhyay, P.; Monticelli, L.; Tieleman, D. P. Molecular dynamics simulation of a palmitoyl-oleoyl phosphatidylserine bilayer with $\mathrm{Na}^{+}$counterions and $\mathrm{NaCl}$. Biophys. J. 2004, 86, 1601-1609.

(27) Kandasamy, S. K.; Larson, R. G. Effect of salt on the interactions of antimicrobial peptides with zwitterionic lipid bilayers. Biochim. Biophys. Acta, Biomembr. 2006, 1758, 1274-1284.

(28) Klauda, J. B.; Venable, R. M.; MacKerell, A. D.; Pastor, R. W. Considerations for lipid force field development. Curr. Top. Membr. 2008, 60, 1-48.

(29) Klauda, J. B.; Venable, R. M.; Freites, J. A.; O'Connor, J. W.; Tobias, D. J.; Mondragon-Ramirez, C.; Vorobyov, I.; MacKerell, A. D.; Pastor, R. W. Update of the CHARMM All-Atom Additive Force Field for Lipids: Validation on Six Lipid Types. J. Phys. Chem. B 2010, 114, $7830-7843$.

(30) Pastor, R. W.; MacKerell, A. D. Development of the CHARMM Force Field for Lipids. J. Phys. Chem. Lett. 2011, 2, 1526-1532.

(31) Piggot, T. J.; Pineiro, A.; Khalid, S. Molecular Dynamics Simulations of Phosphatidylcholine Membranes: A Comparative Force Field Study. J. Chem. Theory Comput. 2012, 8, 4593-4609.

(32) Pluhackova, K.; Kirsch, S. A.; Han, J.; Sun, L. P.; Jiang, Z. Y.; Unruh, T.; Bockmann, R. A. A Critical Comparison of Biomembrane Force Fields: Structure and Dynamics of Model DMPC, POPC, and POPE Bilayers. J. Phys. Chem. B 2016, 120, 3888-3903.

(33) Monje-Galvan, V.; Klauda, J. B. Modeling Yeast Organelle Membranes and How Lipid Diversity Influences Bilayer Properties. Biochemistry 2015, 54, 6852-6861.

(34) Blood, P. D.; Voth, G. A. Direct observation of Bin/amphiphysin/ Rvs (BAR) domain-induced membrane curvature by means of molecular dynamics simulations. Proc. Natl. Acad. Sci. U. S. A. 2006, 103, 15068-15072.

(35) Arkhipov, A.; Yin, Y.; Schulten, K. Four-scale description of membrane sculpting by BAR domains. Biophys. J. 2008, 95, 2806-2821.

(36) Cui, H. S.; Lyman, E.; Voth, G. A. Mechanism of Membrane Curvature Sensing by Amphipathic Helix Containing Proteins. Biophys. J. 2011, 100, 1271-1279.

(37) Hu, M. Y.; Diggins, P.; Deserno, M. Determining the bending modulus of a lipid membrane by simulating buckling. J. Chem. Phys. 2013, 138, 214110.

(38) Sodt, A. J.; Pastor, R. W. Molecular Modeling of Lipid Membrane Curvature Induction by a Peptide: More than Simply Shape. Biophys. J. 2014, 106, 1958-1969.

(39) Tahir, M. A.; Van Lehn, R. C.; Choi, S. H.; Alexander-Katz, A. Solvent-exposed lipid tail protrusions depend on lipid membrane composition and curvature. Biochim. Biophys. Acta, Biomembr. 2016, $1858,1207-1215$.

(40) Wang, X.; Deserno, M. Determining the Lipid Tilt Modulus by Simulating Membrane Buckles. J. Phys. Chem. B 2016, 120, 6061-6073.

(41) Magarkar, A.; Jurkiewicz, P.; Allolio, C.; Hof, M.; Jungwirth, P. Increased Binding of Calcium Ions at Positively Curved Phospholipid Membranes. J. Phys. Chem. Lett. 2017, 8, 518-523. 
(42) Jena, B. P. Intracellular Organelle Dynamics at nm Resolution. Methods Cell Biol. 2008, 90, 19-37.

(43) Marrink, S. J.; Risselada, H. J.; Yefimov, S.; Tieleman, D. P.; de Vries, A. H. The MARTINI force field: Coarse grained model for biomolecular simulations. J. Phys. Chem. B 2007, 111, 7812-7824.

(44) Shinoda, W.; DeVane, R.; Klein, M. L. Zwitterionic Lipid Assemblies: Molecular Dynamics Studies of Monolayers, Bilayers, and Vesicles Using a New Coarse Grain Force Field. J. Phys. Chem. B 2010, $114,6836-6849$.

(45) Orsi, M.; Essex, J. W. The ELBA Force Field for Coarse-Grain Modeling of Lipid Membranes. PLoS One 2011, 6, e28637.

(46) Deserno, M.; Kremer, K.; Paulsen, H.; Peter, C.; Schmid, F. Computational studies of biomembrane systems: theoretical considerations, computer simulation models, and applications. Adv. Polymer Sci. 2014, 260, 237-283.

(47) Müller, M.; Katsov, K.; Schick, M. Biological and synthetic membranes: what can be learned from a coarse-grained description? Phys. Rep. 2006, 434, 113-176.

(48) Reynwar, B. J.; Illya, G.; Harmandaris, V. A.; Muller, M. M.; Kremer, K.; Deserno, M. Aggregation and vesiculation of membrane proteins by curvature-mediated interactions. Nature 2007, 447, 461464.

(49) Marrink, S. J.; Mark, A. E. The mechanism of vesicle fusion as revealed by molecular dynamics simulations. J. Am. Chem. Soc. 2003, 125, 11144-11145.

(50) Risselada, H. J.; Marrink, S. J. Curvature effects on lipid packing and dynamics in liposomes revealed by coarse grained molecular dynamics simulations. Phys. Chem. Chem. Phys. 2009, 11, 2056-2067.

(51) Diggins, P., IV; McDargh, Z. A.; Deserno, M. Curvature Softening and Negative Compressibility of Gel-Phase Lipid Membranes. J. Am. Chem. Soc. 2015, 137, 12752-12755.

(52) Gardner, J. M.; Deserno, M.; Abrams, C. F. Effect of intrinsic curvature and edge tension on the stability of binary mixed-membrane three-junctions. J. Chem. Phys. 2016, 145, 074901.

(53) Vanni, S.; Hirose, H.; Barelli, H.; Antonny, B.; Gautier, R. A sub nanometre view of how membrane curvature and composition modulate lipid packing and protein recruitment. Nat. Commun. 2014, 5, 4916.

(54) Magdeleine, M.; Gautier, R.; Gounon, P.; Barelli, H.; Vanni, S.; Antonny, B. A filter at the entrance of the Golgi that selects vesicles according to size and bulk lipid composition. eLife 2016, 5, e16988.

(55) Pinot, M.; Vanni, S.; Pagnotta, S.; Lacas-Gervais, S.; Payet, L. A.; Ferreira, T.; Gautier, R.; Goud, B.; Antonny, B.; Barelli, H. Polyunsaturated phospholipids facilitate membrane deformation and fission by endocytic proteins. Science 2014, 345, 693-697.

(56) West, A.; Brummel, B. E.; Braun, A. R.; Rhoades, E.; Sachs, J. N. Membrane remodeling and mechanics: Experiments and simulations of alpha-Synuclein. Biochim. Biophys. Acta, Biomembr. 2016, 1858, 15941609.

(57) Kasson, P. M.; Pande, V. S. Control of membrane fusion mechanism by lipid composition: Predictions from ensemble molecular dynamics. PLoS Comput. Biol. 2007, 3, e220.

(58) Kawamoto, S.; Klein, M. L.; Shinoda, W. Coarse-grained molecular dynamics study of membrane fusion: Curvature effects on free energy barriers along the stalk mechanism. J. Chem. Phys. 2015, 143, 243112.

(59) Kawakatsu, T. Statistical Physics of Polymers; Springer: Berlin, 2004.

(60) Matsen, M. W.; Schick, M. Stable and Unstable Phases of a Diblock Copolymer Melt. Phys. Rev. Lett. 1994, 72, 2660-2663.

(61) Drolet, F.; Fredrickson, G. H. Combinatorial screening of complex block copolymer assembly with self-consistent field theory. Phys. Rev. Lett. 1999, 83, 4317-4320.

(62) Balazs, A. C.; Singh, C.; Zhulina, E. Modeling the interactions between polymers and clay surfaces through self-consistent field theory. Macromolecules 1998, 31, 8370-8381.

(63) Dickinson, E.; Pinfield, V. J.; Horne, D. S.; Leermakers, F. A. M. Self-consistent-field modelling of adsorbed casein Interaction between two protein-coated surfaces. J. Chem. Soc., Faraday Trans. 1997, 93, $1785-1790$
(64) Kawakatsu, T.; Kawasaki, K. Hybrid Models for the Dynamics of an Immiscible Binary Mixture with Surfactant Molecules. Phys. A 1990, 167, 690-735.

(65) Kawakatsu, T.; Kawasaki, K.; Furusaka, M.; Okabayashi, H.; Kanaya, T. Late-Stage Dynamics of Phase-Separation Processes of Binary-Mixtures Containing Surfactants. J. Chem. Phys. 1993, 99, 82008217.

(66) Kawakatsu, T.; Kawasaki, K.; Furusaka, M.; Okabayashi, H.; Kanaya, T. Theories and Computer-Simulations of Self-Assembling Surfactant Solutions. J. Phys.: Condens. Matter 1994, 6, 6385-6408.

(67) Laradji, M.; Guo, H.; Zuckermann, M. J. Off-Lattice Monte-Carlo Simulation of Polymer Brushes in Good Solvents. Phys. Rev. E: Stat. Phys., Plasmas, Fluids, Relat. Interdiscip. Top. 1994, 49, 3199-3206.

(68) Daoulas, K. C.; Muller, M. Single chain in mean field simulations: Quasi-instantaneous field approximation and quantitative comparison with Monte Carlo simulations. J. Chem. Phys. 2006, 125, 184904.

(69) Detcheverry, F. A.; Kang, H. M.; Daoulas, K. C.; Muller, M.; Nealey, P. F.; de Pablo, J. J. Monte Carlo simulations of a coarse grain model for block copolymers and nanocomposites. Macromolecules 2008, 41, 4989-5001.

(70) Milano, G.; Kawakatsu, T. Hybrid particle-field molecular dynamics simulations for dense polymer systems. J. Chem. Phys. 2009, 130, 214106.

(71) Milano, G.; Kawakatsu, T. Pressure calculation in hybrid particlefield simulations. J. Chem. Phys. 2010, 133, 214102.

(72) Zhao, Y.; De Nicola, A.; Kawakatsu, T.; Milano, G. Hybrid particle-field molecular dynamics simulations: Parallelization and benchmarks. J. Comput. Chem. 2012, 33, 868-880.

(73) De Nicola, A.; Zhao, Y.; Kawakatsu, T.; Roccatano, D.; Milano, G. Hybrid Particle-Field Coarse-Grained Models for Biological Phospholipids. J. Chem. Theory Comput. 2011, 7, 2947-2962.

(74) De Nicola, A.; Zhao, Y.; Kawakatsu, T.; Roccatano, D.; Milano, G. Validation of a hybrid MD-SCF coarse-grained model for DPPC in nonlamellar phases. Theor. Chem. Acc. 2012, 131, 1167.

(75) De Nicola, A.; Hezaveh, S.; Zhao, Y.; Kawakatsu, T.; Roccatano, D.; Milano, G. Micellar drug nanocarriers and biomembranes: how do they interact? Phys. Chem. Chem. Phys. 2014, 16, 5093-5105.

(76) De Nicola, A.; Kawakatsu, T.; Rosano, C.; Celino, M.; Rocco, M.; Milano, G. Self-Assembly of Triton X-100 in Water Solutions: A Multiscale Simulation Study Linking Mesoscale to Atomistic Models. J. Chem. Theory Comput. 2015, 11, 4959-4971.

(77) Sevink, G. J.; Schmid, F.; Kawakatsu, T.; Milano, G. Combining cell-based hydrodynamics with hybrid particle-field simulations: efficient and realistic simulation of structuring dynamics. Soft Matter 2017, 13, 1594-1623.

(78) Phillips, R.; Quake, S. R. The biological frontier of physics. Phys. Today 2006, 59, 38-43.

(79) Jensen, G. V.; Lund, R.; Gummel, J.; Narayanan, T.; Pedersen, J. S. Monitoring the transition from spherical to polymer-like surfactant micelles using small-angle X-ray scattering. Angew. Chem., Int. Ed. 2014, $53,11524-11528$.

(80) Nickels, J. D.; Chatterjee, S.; Stanley, C. B.; Qian, S.; Cheng, X.; Myles, D. A. A.; Standaert, R. F.; Elkins, J. G.; Katsaras, J. The in vivo structure of biological membranes and evidence for lipid domains. PLoS Biol. 2017, 15, e2002214.

(81) Zhu, Y. L.; Lu, Z. Y.; Milano, G.; Shi, A. C.; Sun, Z. Y. Hybrid particle-field molecular dynamics simulation for polyelectrolyte systems. Phys. Chem. Chem. Phys. 2016, 18, 9799-9808.

(82) Zhu, Y. L.; Liu, H.; Li, Z. W.; Qian, H. J.; Milano, G.; Lu, Z. Y. GALAMOST: GPU-accelerated large-scale molecular simulation toolkit. J. Comput. Chem. 2013, 34, 2197-2211.

(83) Cascella, M.; Neri, M. A.; Carloni, P.; Dal Peraro, M. Topologically based multipolar reconstruction of electrostatic interactions in multiscale simulations of proteins. J. Chem. Theory Comput. 2008, 4, 1378-1385.

(84) Alemani, D.; Collu, F.; Cascella, M.; Dal Peraro, M. A Nonradial Coarse-Grained Potential for Proteins Produces Naturally Stable Secondary Structure Elements. J. Chem. Theory Comput. 2010, 6, 315-324. 
(85) Spiga, E.; Alemani, D.; Degiacomi, M. T.; Cascella, M.; Dal Peraro, M. Electrostatic-Consistent Coarse-Grained Potentials for Molecular Simulations of Proteins. J. Chem. Theory Comput. 2013, 9, $3515-3526$

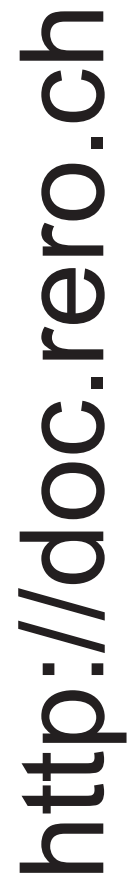

